\title{
Erkrankungszahlen in der Pneumologie - eine Projektion bis 2060
}

\author{
Disease Numbers in Pneumology - A Projection to 2060
}

Autoren

Institute
R. Pritzkuleit ${ }^{1}$, F. Beske², A. Katalinic ${ }^{1}$

Institut für Krebsepidemiologie an der Universität zu Lübeck

2 Institut für Gesundheits-System-Forschung, Kiel eingereicht 1.7.2010

akzeptiert 5.7.2010

Bibliografie

DOI http://dx.doi.org/ 10.1055/s-0030-1255637

Pneumologie 2010; 64:

535-540 @ Georg Thieme Verlag KG Stuttgart · New York ISSN 0934-8387

\section{Korrespondenzadresse} Dr. Ron Pritzkuleit

Institut für Krebsepidemiologie Ratzeburger Allee 160

23538 Lübeck

Ron.Pritzkuleit@uk-sh.de

\section{Zusammenfassung \\ $\nabla$}

Hintergrund: Der demografische Wandel führt zu einer Veränderung der Alterszusammensetzung der Bevölkerung. Mit einer Status quo-Projektion werden für fünf Erkrankungsbilder der Lunge (COPD, CAP, Lungenkrebs, Asthma bronchiale und Tuberkulose) die Fallzahlen für Deutschland bis 2060 geschätzt.

Material und Methoden: Auf Basis der 12. koordinierten Bevölkerungsvorausberechnung des Statistischen Bundesamtes werden alters- und geschlechtsspezifische Inzidenz- bzw. Prävalenzraten auf die erwartete Bevölkerung übertragen. Die beschriebenen Entwicklungen beruhen ausschließlich auf demografischen Veränderungen.

Ergebnisse: Die absoluten Fallzahlen für Asthma bronchiale und Tuberkulose werden leicht sinken, bei COPD und Lungenkrebs sind zunächst ansteigende, später sinkende Fallzahlen zu erwarten und bei CAP sind große Steigerungen wahrscheinlich. Wegen der sinkenden Bevölkerungszahl werden die Raten (Krankheitslast der Bevölkerung) deutlich steigen.

Diskussion: Ursachen des demografischen Wandels sind steigende Lebenserwartung, konstant niedrige Geburtenraten und der Eintritt der geburtenstarken Jahrgänge in die Lebensphase erhöhten Krankheitsrisikos. Die steigende Belastung des Gesundheitssystems, auch durch Erkrankungen der Lunge, erfordert eine Diskussion um die Priorisierung von Gesundheitsleistungen.

\section{Einleitung}

\section{$\nabla$}

In den nächsten Jahrzehnten wird sich in Deutschland, ebenso wie in den meisten Industriestaaten, eine Veränderung der Alterszusammensetzung der Bevölkerung vollziehen [1] . Die Zahl der Menschen, die älter als 65 Jahre sind, wird von heute 16,8 Millionen auf 22,0 Millionen im Jahr 2060 steigen. Gleichzeitig sinkt die Ge-

\section{Abstract \\ $\nabla$}

Background: The demographic change leads to a change in the age-composition of the population. We have calculated a status quo projection of the absolute numbers for five diagnoses of the lung (COPD, CAP, lung cancer, bronchial asthma and tuberculosis) for Germany up to 2060.

Material and Methods: Based on the $12^{\text {th }}$ coordinated population prediction of the Federal Statistics Office, we transferred age- and sex-specific incidence and prevalence rates, respectively, to the expected population. All described developments are based solely on demographic changes. Results: The absolute numbers of bronchial asthma and tuberculosis will experience a minor decrease. We expect at first increasing and later decreasing case numbers for COPD and lung cancer. A major increase of the case numbers for CAP will be probable. By reason of a decreasing population, the rates (burden of disease for the population) will increase considerably.

Discussion: The demographic change is mainly caused by increasing life expectancy, constantly low birth rates, and the entry of the baby-boom generation into the age of higher disease risks. A discussion about prioritisation of health care is needed because of the rising burdens for the health system, including diseases of the lung. 
dass in den kommenden Jahrzehnten die absoluten Erkrankungszahlen zunehmen werden. Steigende Erkrankungszahlen bei zugleich abnehmender Gesamtbevölkerung führen dann zu einer nochmals stärker ansteigenden Krankheitslast in der Bevölkerung.

Die genaue Vorhersage von Erkrankungszahlen über einen langen Zeitraum von 50 Jahren ist seriös nicht möglich. Zu vielfältig sind die zukünftigen Einflussfaktoren - wissenschaftlich-technische Neuerungen beeinflussen Therapie und Diagnostik, sozioökonomische Verhältnisse sind eng mit Lebensstilfaktoren verbunden, die ihrerseits wiederum einen Einfluss auf das Erkrankungsrisiko haben können, sich ändernde Umweltbedingungen beeinflussen das Erkrankungsrisiko und politische Rahmenbedingungen schlagen sich in der medizinischen Versorgungssituation nieder. Zwischen den genannten und einer Reihe weiterer Faktoren bestehen darüber hinaus Interdependenzen, die kaum abgeschätzt werden können. Auch wenn Szenarienmodelle denkbar sind [2], so wie bei Beelte et al. altersspezifische Trends der Lungenkrebsinzidenz in Folge geänderten Rauchverhaltens bis 2020 modelliert wurden [3], sind die Grundannahmen kritisch zu hinterfragen.

Während sehr langfristige Prognosen im Sinne einer Vorhersage also kaum möglich und sinnvoll erscheinen, ist eine Projektion der jetzigen Morbiditätsverhältnisse auf zukünftige Bevölkerungen sinnvoll. Damit wird ein Denkmodell entwickelt, das ceteris paribus den Einfluss der demografischen Entwicklung auf zukünftige mögliche Erkrankungszahlen abbildet. Ein Beispiel für eine solche Projektion für das Jahr 2050 findet sich bei Peters et al. [4] und bei Katalinic et al. [5].

Ziel der vorliegenden Arbeit ist die Deskription des Einflusses der sich ändernden demografischen Verhältnisse auf Erkrankungen der Lunge.

\section{Exkurs Demographie}

Für die sich abzeichnende demografische Entwicklung sind im Wesentlichen drei Faktoren verantwortlich - eine seit ca. 35 Jahren anhaltend niedrige Geburtenrate, eine relativ hohe Geburtenrate in der Zeit zwischen 1955 und 1970 sowie eine stetig steigende Lebenserwartung. Außerdem ist zu berücksichtigen, dass demografische Prozesse langfristige Auswirkungen haben. Ein geborenes Kind beeinflusst die Demografie die folgenden 70 bis 80 Jahre, die ältere Generation von 2060 ist heute bereits geboren und damit zahlenmäßig schon weitgehend festgelegt.

Die Geburtenrate liegt, mit nur sehr geringen Schwankungen, seit Mitte der 1970er-Jahre (zumindest auf dem Gebiet der früheren BRD) bei etwa 1,4 Kindern je Frau. Um den zahlenmäßigen Ersatz einer Generation durch die nachfolgende Generation sicherzustellen, wäre eine Geburtenrate von rund 2,1 Kindern je Frau notwendig. Dieser Wert wurde auf dem Gebiet der früheren Bundesrepublik das letzte Mal im Jahre 1969 (1969: 2,21, 1970: 2,01) und auf dem Gebiet der ehemaligen DDR im Jahre 1971 (1971: 2,13, 1972: 1,79) erreicht. Seit dieser Zeit ist die nachfolgende Generation zahlenmäßig immer kleiner als die Vorgängergeneration.

Zwischen 1955 und 1970 lag die Geburtenrate jeweils über 2 Kindern je Frau (höchster Wert 1964 - BRD: 2,54; DDR: 2,51; Angaben Statistisches Bundesamt). Diese Kohorte der sogenannten geburtenstarken Jahrgänge „schiebt“ sich als Berg durch die Bevölkerungspyramide Deutschlands. Im Jahre 2029 erreicht der Jahrgang der damals höchsten Geburtenrate das Alter von 65 Jah- ren und 2035 wird die gesamte Kohorte diese Altersgrenze überschritten haben. Das Phänomen der hohen Personenzahl der geburtenstarken Jahrgänge ist nicht neu und stand bereits mehrfach im öffentlichen Interesse (z.B. Schuleintritt der Kohorte). Ab etwa 2020 wird die Kohorte für das Gesundheitswesen relevant.

Die durchschnittliche Lebenserwartung liegt nach der aktuellen Sterbetafel (2006/2008) [6] bei 74,04 Jahre für neugeborene Jungen und 80,27 Jahre für neugeborene Mädchen. Damit hat sich die Lebenserwartung bei Geburt in zehn Jahren um 3,13 Jahre $(+4,2 \%)$ für Jungen und 2,13 Jahre $(+2,7 \%)$ für Mädchen erhöht. Von größerem Interesse als die Lebenserwartung bei Geburt diese Personen treten erst 2075 in das Alter erhöhten Krankheitsrisikos ein - ist die fernere Lebenserwartung bereits Geborener. Die fernere Lebenserwartung einer Person aus den geburtenstarken Jahrgängen (45 Jahre in der aktuellen Sterbetafel) stieg um 7,9\% (2,46 Jahre) bei Männern und 4,9\% (1,78 Jahre) bei Frauen (alle Vergleiche gegenüber Sterbetafel 1996/1998 [7]).

Die beschriebenen drei Effekte überlagern einander. Einer ohnehin von Generation zu Generation stetig sinkenden Bevölkerungszahl steht die zahlenmäßig starke Gruppe der geburtenstarken Jahrgänge gegenüber, die ihrerseits eine höhere Lebenserwartung hat als vorhergehende Generationen. In $\bullet$ Tab. 1 sind wichtige Kennzahlen der erwarteten Bevölkerungsentwicklung aufgeführt. Dazu zählt auch das zahlenmäßige Verhältnis zwischen der Bevölkerung im arbeitsfähigen Alter und der älteren Bevölkerungsgruppe, die ein erhöhtes Erkrankungsrisiko hat. Dieses Verhältnis (Altenquotient) wird sich nahezu halbieren, von 2,9 auf 1,5. Auch wenn man berücksichtigt, dass an der Basis der Bevölkerungspyramide durch die geringere Zahl an Geburten die Belastungen der Bevölkerung im arbeitsfähigen Alter abnimmt, so steigt die Gesamtbelastung der 20- bis 65-Jährigen von 1,6 auf 1,0 im Jahre 2060. Das heißt einer Person im arbeitsfähigen Alter steht dann eine Person im nicht arbeitsfähigen Alter gegenüber [8].

\section{Methoden}

$\nabla$

Für die Projektion wurden bevölkerungsbezogene alters- und geschlechtsspezifische Inzidenz- bzw. Prävalenzdaten verwendet. Diese wurden auf die entsprechenden Altersgruppen der 12. koordinierten Bevölkerungsvorausberechnung für Deutschland des Statistischen Bundesamtes übertragen. Die Summe über die in allen Altersgruppen berechnete Fallzahl ergibt die Gesamtfallzahl für das jeweilige Jahr. Zur Abschätzung der Krankheitslast in der Bevölkerung wurden die entsprechenden rohen Raten ermittelt. Als Bevölkerungsdaten wurde ausschließlich die Variante 1-W1 („mittlere Bevölkerung, Untergrenze“) verwandt [8]. Auf die Berechnung mit unterschiedlichen Varianten der 12. koordinierten Bevölkerungsvorausberechnung wurde bewusst verzichtet. Ziel der Arbeit ist das Aufzeigen eines Trends, nicht die möglichst genaue Vorhersage der Fallzahlen. Dazu ist eine mittlere Variante der Bevölkerungsvorausberechnung ausreichend. Diese Variante unterstellt eine konstante Geburtenhäufigkeit von 1,4 Kindern pro Frau, einen allmählich ansteigenden positiven Wanderungssaldo auf 100000 Personen jährlich bis zum Jahr 2014 (ab dann konstant) und eine moderate Steigerung der Lebenserwartung auf 85,0 Jahre bei neugeborenen Jungen und 89,2 Jahre bei neugeborenen Mädchen im Jahre 2060.

Die ausgewählten Krankheiten für den Bereich der Lungenkrankheiten sind als Tracerdiagnosen zu verstehen. Ausgewählt wurde 


\begin{tabular}{|c|c|c|c|c|}
\hline \multirow[b]{2}{*}{ Bevölkerung in Mio. } & \multirow{2}{*}{$\begin{array}{l}2010 \\
81,5\end{array}$} & \multirow{2}{*}{$\begin{array}{l}2030 \\
77,4 \\
(-5,1 \%)\end{array}$} & \multirow{2}{*}{$\begin{array}{l}2050 \\
69,4 \\
(-14,9 \%)\end{array}$} & \multirow{2}{*}{$\begin{array}{l}2060 \\
64,7 \\
(-20,7 \%)\end{array}$} \\
\hline & & & & \\
\hline $\begin{array}{l}\text { Bevölkerung unter } 20 \text { Jahre } \\
\text { in Mio. }\end{array}$ & 15,0 & $\begin{array}{l}12,9 \\
(-13,9 \%)\end{array}$ & $\begin{array}{l}10,7 \\
(-28,7 \%)\end{array}$ & $\begin{array}{l}10,1 \\
(-32,8 \%)\end{array}$ \\
\hline $\begin{array}{l}\text { Bevölkerung zwischen } 20 \text { und } 65 \text { Jahre } \\
\text { in Mio. }\end{array}$ & 49,7 & $\begin{array}{l}42,1 \\
(-15,2 \%)\end{array}$ & $\begin{array}{l}35,7 \\
(-28,2 \%)\end{array}$ & $\begin{array}{l}32,6 \\
(-34,5 \%)\end{array}$ \\
\hline $\begin{array}{l}\text { Bevölkerung } 65 \text { Jahre und älter } \\
\text { in Mio. }\end{array}$ & 16,8 & $\begin{array}{l}22,3 \\
(+32,6 \%)\end{array}$ & $\begin{array}{l}23,0 \\
(+36,8 \%)\end{array}$ & $\begin{array}{l}22,0 \\
(+30,8 \%)\end{array}$ \\
\hline $\begin{array}{l}\text { Anteil der Personen } 65 \text { Jahre und älter } \\
\text { an der Gesamtbevölkerung }\end{array}$ & $20,6 \%$ & $28,8 \%$ & $33,1 \%$ & $34,0 \%$ \\
\hline $\begin{array}{l}\text { Personen im arbeitsfähigen Alter } \\
\text { je Person im Rentenalter } \# \$ \\
\text { (Altenquotient) }\end{array}$ & 2,9 & 1,9 & 1,6 & 1,5 \\
\hline $\begin{array}{l}\text { Personen im arbeitsfähigen Alter } \\
\text { je Person im nicht arbeitsfähigen Alter \#\& } \\
\text { (Belastungsquotient) }\end{array}$ & 1,6 & 1,2 & 1,1 & 1,0 \\
\hline $\begin{array}{l}\text { Quelle: Statistisches Bundesamt, 12. koordin } \\
\text { Untergrenze) [8] } \\
\text { Prozentangaben = Abweichung gegenüber } 2 \\
\text { "Personen im arbeitsfähigen Alter = Persone } \\
\text { \$Personen im Rentenalter = Personen } 65 \text { Jahr }\end{array}$ & $\begin{array}{l}\text { hen } 20 \text { bi } \\
\text { ter }\end{array}$ & $\begin{array}{l}\text { erechnung, } \\
\text { ahre }\end{array}$ & & Bevölkerung, \\
\hline
\end{tabular}

Tab. 1 Kennzahlen der demografischen Entwicklung.

\begin{tabular}{|c|c|c|c|c|}
\hline & 2010 & 2030 & 2050 & 2060 \\
\hline \multicolumn{5}{|l|}{ Asthma bronchiale (Prävalenz) } \\
\hline Fallzahl (Prävalenz) & 4,6 Mio. & 4,4 Mio. & 4,0 Mio. & 3,8 Mio. \\
\hline Rohe Rate pro 100000 & 5691 & 5743 & 5833 & 5817 \\
\hline \multicolumn{5}{|l|}{ Tuberkulose (Inzidenz pro Jahr) } \\
\hline Fallzahl (Inzidenz pro Jahr) & 4600 & 4600 & 4300 & 4000 \\
\hline Rohe Rate pro 100000 & 5,6 & 5,9 & 6,3 & 6,2 \\
\hline \multicolumn{5}{|l|}{ COPD (Prävalenz) } \\
\hline Fallzahl (Prävalenz) & 6,8 Mio. & 7,9 Mio. & 8,0 Mio. & 7,5 Mio. \\
\hline Rohe Rate pro 100000 & 8305 & 10271 & 11475 & 11561 \\
\hline \multicolumn{5}{|l|}{ Lungenkrebs (Inzidenz pro Jahr) } \\
\hline Fallzahl & 50000 & 61000 & 59000 & 56000 \\
\hline Rohe Rate pro 100000 & 61,4 & 78,9 & 85,5 & 86,5 \\
\hline \multicolumn{5}{|l|}{ CAP (Inzidenz pro Jahr) } \\
\hline Fallzahl & 1,2 Mio. & 2,0 Mio. & 2,9 Mio. & 2,8 Mio. \\
\hline Rohe Rate pro 100000 & 1519 & 2566 & 4240 & 4375 \\
\hline
\end{tabular}

Tab. 2 Projizierte Fallzahlen und rohe Raten bis 2060
COPD, die zugehörigen altersspezifischen Prävalenzdaten entstammen der Publikation von Geldmacher et al. [9]. Für die ambulant erworbene Pneumonie (CAP - Community Acquired Pneumonia) wurden nicht veröffentlichte Inzidenzdaten aus dem Projekt Capnetz ( www.capnetz.de) verwendet, die Bestimmung der Inzidenz ist aber schwierig und wird bei Schnoor et al. [10] beschrieben. Die Auswertungen zur Tuberkulose basieren auf Inzidenzdaten, publiziert vom Robert Koch-Institut im Bericht zur Epidemiologie der Tuberkulose in Deutschland 2008 [11] (Rohdaten wurden vom Herausgeber freundlicherweise zur Verfügung gestellt). Ebenfalls aus einer Publikation des Robert Koch-Instituts entstammen die Angaben zur Prävalenz des Asthma bronchiale [12]. Die Angaben basieren auf der zweiten Welle des telefonischen Gesundheitssurveys. Quelle für die Inzidenz des Lungenkrebses ist die Gesellschaft der epidemiologischen Krebsregister in Deutschland (GEKID), die die Daten im Rahmen einer Deutschlandschätzung online zur Verfügung stellt [www. gekid.de].

\section{Ergebnisse}

Drei Maßzahlen sind für die Bewertung des Effekts des demografischen Wandels auf die entsprechende Erkrankung von Bedeutung - die absolute Fallzahl, die rohe Rate (siehe $\bullet$ Tab. 2) sowie die prozentuale Veränderung gegenüber dem Jahr 2010 (siehe - Abb.1). Die Prävalenzen des Asthma bronchiale und der COPD sind mit rund fünf bzw. rund sieben Millionen Erkrankten bereits heute sehr hoch. Bis 2060 zeigt sich ein prozentualer Rückgang der absoluten Fallzahlen gegenüber 2010 für Asthma um $18,9 \%$, während die bevölkerungsbezogene Rate sich relativ nur wenig ändert. Für die Tuberkulose deutet sich ein Rückgang der Erkrankungszahlen an, die bevölkerungsbezogene Erkrankungsrate 2060 liegt aber etwa 10\% höher als heute.

Bei der COPD liegt die Anzahl der Erkrankungen im Jahr 2040 um etwa 22,7\% höher als 2010, danach verringert sich der Abstand zu 2010 wieder etwas. Durch den Rückgang der Bevölkerung steigen die populationsbezogenen Raten ab 2040 aber weiter, wenn auch nur noch dezent, an. Lungenkrebs zeigt ein ähnliches Bild wie die COPD.

Bei der ambulant erworbenen Lungenentzündung steigt die Erkrankungsrate bis 2060 kontinuierlich (bis zu 188\% gegenüber 2010) an. 


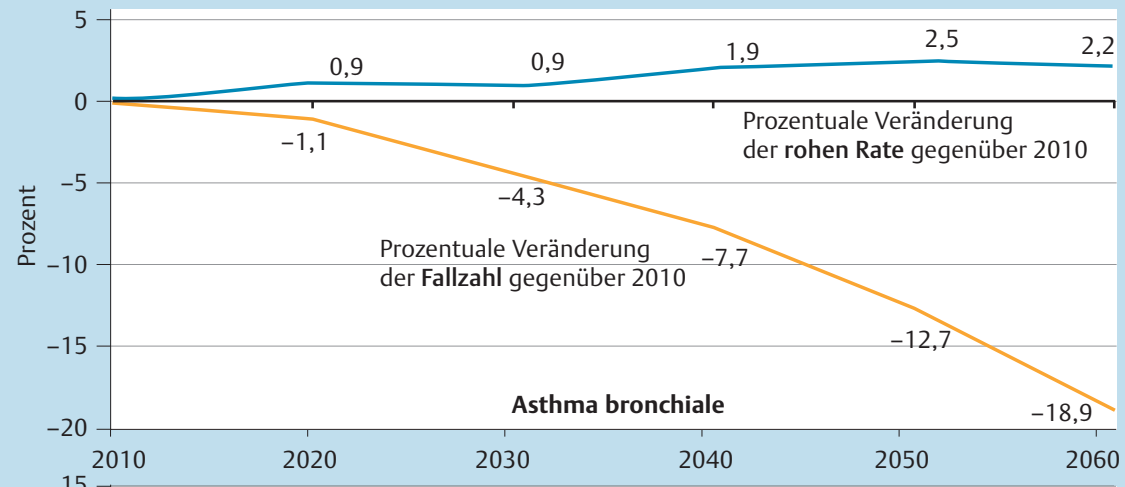

Abb. 1 Prozentuale Veränderungen der Erkrankungszahlen (gelb) und der rohen Raten (blau) gegenüber 2010 .
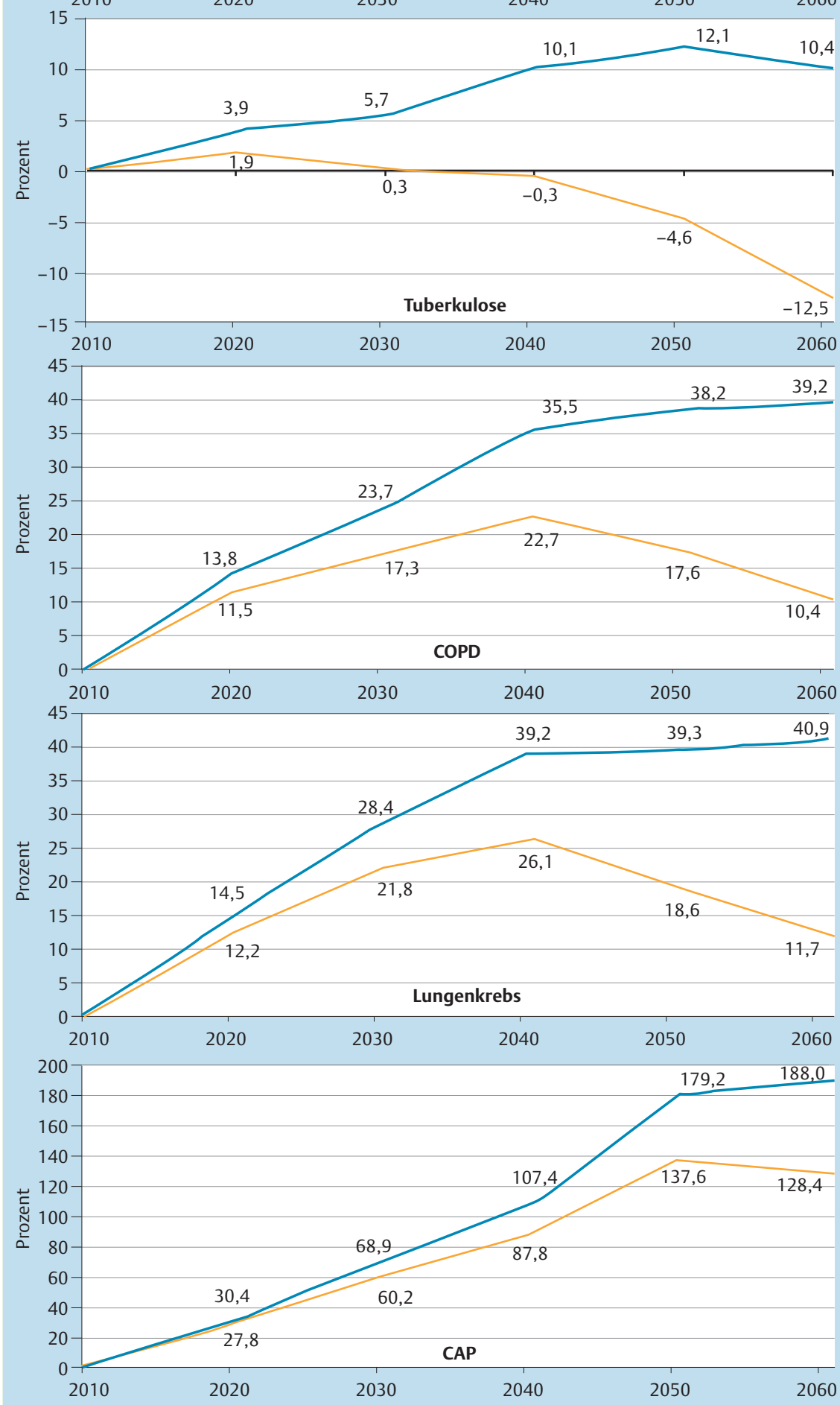


\section{Diskussion}

\section{V}

Ziel der Arbeit war die Projektion von Fallzahlen von Krankheiten der Lunge in das Jahr 2060. Dabei wurde ein ceteris paribus-Ansatz gewählt, der die Krankheitshäufigkeit auf dem aktuellen Stand konstant hält bei gleichzeitig variabler Bevölkerungsentwicklung. Alle dargestellten Ergebnisse sind demnach direkte Folge der demografischen Entwicklung in Deutschland. Einflüsse aus der Wissenschaft (Entwicklung neuer Diagnosen und Therapien), Veränderungen in den sozialen und politischen Rahmenbedingungen sowie von Risikofaktoren oder Umweltbedingungen wurden vernachlässigt bzw. als unverändert zu den heutigen Zuständen unterstellt. Die durchgeführte Projektion stellt somit ein Denkmodell dar, das den Einfluss der sich ändernden demografischen Verhältnisse auf die Morbidität der Bevölkerung beschreibt.

Es zeigt sich, dass die Fallzahlen für die betrachteten Krankheitsbilder in den nächsten Jahrzehnten relevant ansteigen werden. Der Anstieg erfolgt allerdings für die betrachteten Erkrankungen unterschiedlich. Die höchsten absoluten Erkrankungszahlen stellen sich zwischen 2030 und 2050 ein. Das ist gerade die Periode, in der die Bevölkerung der geburtenstarken Jahrgänge in die Lebensphase erhöhten Erkrankungsrisikos eintritt. Die deutlich gestiegene absolute Bevölkerungszahl in der Altersklasse der dann 60 - bis 80 -Jährigen führt unweigerlich zu einer höheren absoluten Erkrankungszahl. Gesondert zu betrachten ist die Entwicklung der rohen Erkrankungsraten. Die können als Krankheitslast der Bevölkerung interpretiert werden. Sie wird in den nächsten Jahrzehnten ansteigen und ab dem Jahr 2050 nahezu konstant bleiben bzw. nur in geringerem Maße wieder absinken, da die Bevölkerungszahl in Deutschland ebenfalls abnimmt. Wenn die Bevölkerungszahl stärker sinkt als die Erkrankungszahlen (ab 2050), führt dies trotzdem zu weiter steigenden rohen Raten und damit zu einer weiter steigenden Krankheitslast.

Die fünf ausgewählten Krankheitsbilder aus dem Bereich der Pneumologie zeigen unterschiedliche Entwicklungen. Für Tuberkulose und Asthma bronchiale ist ceteris paribus mit sinkenden Fallzahlen zu rechnen. Das liegt an dem geringen Altersgradienten dieser beiden Krankheitsbilder. Die Epidemiologie des Asthma bronchiale weist höhere Prävalenzraten in jüngeren Altersgruppen aus [12], der Altersgang bei der Tuberkulose zeigt bei Männern relativ konstante Inzidenzraten zwischen dem 25. und 70. Lebensjahr (danach steigend) und bei Frauen zwei Erkrankungsgipfel - in der Altersgruppe der 25- bis 29-Jährigen und ab dem 70. Lebensjahr. Die drei anderen Krankheitsbilder zeigen dagegen einen deutlichen Anstieg des Erkrankungsrisikos in den höheren Altersgruppen, sodass für diese die Verschiebung des Altersspektrums der Bevölkerung stärker zum Tragen kommt. Drei Gründe können aus demografischer Sicht für die Entwicklung der Morbidität benannt werden - eine konstant niedrige Geburtenrate mit der damit verbundenen konstanten zahlenmäßigen Verkleinerung der Bevölkerung, eine stetig steigende Lebenserwartung der Bevölkerung und das Durchlaufen der Bevölkerungspyramide der Kohorte der geburtenstarken Jahrgänge. Der letztgenannte Grund ist in seiner zeitlichen Dimension genau zu beschreiben. Im Jahre 2060 wird der letzte Jahrgang (1970) der geburtenstarken Jahrgänge das 90. Lebensjahr vollenden und stirbt damit sukzessive aus. Bis dahin ist es aber notwendig, die medizinische Infrastruktur den vorher steigenden Erkrankungszahlen entsprechend anzupassen. Die Steigerung der Fallzahlen um 23\% bei der COPD bzw. um 26\% beim Lungenkrebs (2040 gegenüber 2010, vgl. Abb. 1) bedeutet für 2040 etwa ein Mehr von 1,6 Millionen bzw. 13000 Therapien als 2010. Im Falle der ambulant erworbenen Lungenentzündung ist die Steigerung bis 2050 mit $+138 \%$ (+ 1,7 Millionen) sogar noch höher. Dagegen ist mit freiwerdenden Ressourcen beim Asthma bronchiale $(-13 \%=-600000)$ und bei Tuberkulose $(-5 \%=-200)$ zu rechnen. Damit wird sich auch das Diagnose- und Therapiespektrum im Bereich der Lungenerkrankungen verschieben. Ab 2050 beginnt die Alterung der geburtenstarken Jahrgänge als demografischer Einfluss auf die Morbidität der Bevölkerung deutlich abzunehmen.

Aber werden die Ergebnisse auch genauso eintreten, wie hier prognostiziert? Sicher nicht genauso, dazu ist die Reduzierung der durchgeführten Projektion allein auf demografische Effekte zu wenig spezifisch. Außerdem, so ist zu hoffen, führen solche Projektionen zu weiteren Anstrengungen in Prävention und Forschung, um den gezeigten Entwicklungen entgegenzuwirken. Gerade deshalb ist das Aufzeigen des möglichen bedeutsamen Einflusses des demografischen Wandels auf die Häufigkeit von Erkrankungen mittels einer Projektion von großer Relevanz und gerechtfertigt.

Kritisch muss auch die Auswahl der Inzidenz- bzw. Prävalenzdaten diskutiert werden. Bei der Tuberkulose zum Beispiel wäre eine Adjustierung nach Nationalität notwendig. Die Inzidenz zwischen Einwohnern deutscher und ausländischer Nationalität unterscheidet sich um den Faktor 5,4 (3,9 zu 20,7; Gesamtinzidenz 5,5; [11]) mit der höheren Inzidenz bei Ausländern. Eine solche Adjustierung ist mit den vorliegenden Daten der Bevölkerungsvorausschätzung aber nicht möglich. Unsere Analyse unterstellt somit einen gleichbleibenden Ausländeranteil mit jeweils konstanten altersspezifischen Erkrankungswahrscheinlichkeiten. Bei der ambulant erworbenen Pneumonie ist die Inzidenzbestimmung an sich mit großen Unsicherheiten verbunden [10], was dann in stärkerem Maße auch für die altersspezifischen Inzidenzraten gilt. Trotz aller Einschränkungen bei den Morbiditätsdaten bietet die Projektion insgesamt Hinweise auf die Trendentwicklung der Erkrankungszahlen im Bereich der Lungenkrankheiten.

\section{Fazit}

\section{$\nabla$}

Die konstant niedrigen Geburtenraten in Deutschland, die stetig steigende Lebenserwartung und vor allem das Altern der Geburtskohorte 1955-1970 werden die Morbidität der Bevölkerung in Deutschland in den nächsten 50 Jahren beeinflussen. Für den Bereich der Lungenerkrankungen ist bei der COPD, bei der ambulant erworbenen Pneumonie und beim Lungenkrebs mit stetig steigenden absoluten Fallzahlen zu rechnen. Diese Entwicklung dürfte sich ab dem Jahr 2050 etwas entspannen. Die Krankheitslast der Bevölkerung für Lungenerkrankungen, gemessen als Erkrankungsrate, wird trotz teilweise leicht rückgängiger absoluter Erkrankungszahlen wegen der insgesamt abnehmenden Bevölkerungszahl weiter steigen.

Nicht nur für Erkrankungen der Lunge ist mit steigenden Fallzahlen in den nächsten Jahrzehnten zu rechnen [4,5], vielmehr werden nahezu alle Bereiche der künftigen Krankheitsversorgung betroffen sein. Es stellt sich die Frage nach der Finanzierbarkeit dieser Entwicklung. Bei steigenden Belastungen des Gesundheitssystems und abnehmender Zahl einzahlender Versicherter wird eine Diskussion um die Priorisierung von Gesundheitsleistungen notwendig sein. 


\section{Interessenkonflikte}

Die Autor geben an, dass kein Interessenkonflikt besteht.

\section{Literatur}

1 Christensen K, Doblhammer G, Rau $R$ et al. Ageing populations: the challange ahead. Lancet 2009; 374: 1196-1208

2 Nowossadeck E. Morbiditätsprognosen auf Basis von Bevölkerungsprognosen. Welchen Beitrag kann ein Gesundheitsmonitoring leisten? Bundesgesundheitsblatt Gesundheitsforschung Gesundheitsschutz 2010; 53: 427-434

3 Beelte A-K, Pritzkuleit R, Katalinic A. Lungenkrebsinzidenz und -mortalität - aktuelle Trends und Hochrechnungen aus dem Krebsregister Schleswig-Holstein. Dtsch med Wochenschr 2008; 133: 1487-1492

4 Peters E, Pritzkuleit R, Beske F et al. Demographischer Wandel und Krankheitshäufigkeiten. Eine Projektion bis 2050. Bundesgesundheitsblatt Gesundheitsforschung Gesundheitsschutz 2010; 53: 417-426

5 Katalinic A, Peters E, Beske F et al. Projection of morbidity 2030 and 2050: impact for the National Health System and blood supply. Transfus Med Hemother 2010; 37: 155 - 159
6 Statistisches Bundesamt. Bevölkerung und Erwerbstätigkeit. Sterbetafel Deutschland 2006/2008. Wiesbaden: 2009

7 Statistisches Bundesamt. Periodensterbetafeln für Deutschland. Allgemeine und abgekürzte Sterbetafeln 1871/1881 bis 2003/2005. Wiesbaden: 2006

8 Statistisches Bundesamt. Bevölkerung Deutschlands bis 2060. Ergebnisse der 12. koordinierten Bevölkerungsvorausberechnung. Wiesbaden: 2009

9 Geldmacher H, Biller H, Herbst A et al. Die Prävalenz der chronisch obstruktiven Lungenerkrankung (COPD) in Deutschland. Dtsch med Wochenschr 2008; 133: 2609-2614

10 Schnoor M, Hedicke J, Dalhoff K et al. Appoaches to estimate the population-based incidence of community acquired pneumonia. J Infect 2007; 55: 233-239

11 Robert Koch-Institut (Hrsg). Bericht zur Epidemiologie der Tuberkulose in Deutschland für 2008. Berlin: 2010

12 Robert Koch-Institut (Hrsg). Telefonischer Gesundheitssurvey des Robert Koch-Instituts (2. Welle). Deskriptiver Ergebnisbericht. Beiträge zur Gesundheitsberichterstattung. Berlin: 2006 